\title{
Polyspecies biofilm formation on implant surfaces with different surface characteristics
}

\author{
Patrick R. SCHMIDLIN 1 , Phillip MÜLLER², Thomas ATTIN ${ }^{3}$, Marco WIELAND ${ }^{4}$, Deborah HOFER ${ }^{5}$, Bernhard \\ GUGGENHEIM ${ }^{6}$
}

\begin{abstract}
1- PD Dr. med. dent., Head of Periodontology, Clinic of Preventive Dentistry, Periodontology and Cariology, Center of Dental Medicine, University of Zurich, Zurich, Switzerland

2- Dr. med. dent., Research Assistant, Clinic of Preventive Dentistry, Periodontology and Cariology, Center of Dental Medicine, University of Zurich, Zurich, Switzerland.

3- Prof. Dr. med. dent., Chairman, Clinic of Preventive Dentistry, Periodontology and Cariology, Center of Dental Medicine, University of Zurich, Zurich, Switzerland. 4- PhD, MyoPowers Medical Technologies SA, Lausanne, Switzerland before Institute Straumann AG, Basel, Switzerland.

5- RDH, BS, EdM, CAS, Research Associate, Clinic of Preventive Dentistry, Periodontology and Cariology, Center of Dental Medicine, University of Zurich, Zurich, Switzerland.

6- Prof. em., Former Head of Department, Institute for Oral Biology, Section for Oral Microbiology and General Immunology, Center of Dental Medicine, University of Zurich, Zurich, Switzerland.
\end{abstract}

Corresponding address: Patrick R. Schmidlin - Center of Dental Medicine, Plattenstrasse 11, 8032 - Zurich, Switzerland - Phone: +41 446343417 - Fax +4144634 4308 - e-mail: patrick.schmidlin@zzm.uzh.ch

Submitted: April 17, 2012 - Modified: November 22, 2012 - Accepted: January 3, 2013

\section{ABSTRACT}

\begin{abstract}
$\mathrm{O}$ bjective: To investigate the microbial adherence and colonization of a polyspecies biofilm on 7 differently processed titanium surfaces. Material and Methods: Six-species biofilms were formed anaerobically on 5-mm-diameter sterilized, saliva-preconditioned titanium discs. Material surfaces used were either machined, stained, acid-etched or sandblasted/ acid-etched (SLA). Samples of the latter two materials were also provided in a chemically modified form, with increased wettability characteristics. Surface roughness and contact angles of all materials were determined. The discs were then incubated anaerobically for up to $16.5 \mathrm{~h}$. Initial microbial adherence was evaluated after 20 min incubation and further colonization after 2, 4, 8, and 16.5 h using non-selective and selective culture techniques. Results at different time points were compared using ANOVA and Scheffé post hoc analysis. Results: The mean differences in microorganisms colonizing after the first 20 min were in a very narrow range (4.5 to $4.8 \mathrm{log}$ CFU). At up to $16.5 \mathrm{~h}$, the modified SLA surface exhibited the highest values for colonization $(6.9 \pm 0.2 \mathrm{log} C F U, p<0.05)$ but increasing growth was observed on all test surfaces over time. Discrepancies among bacterial strains on the differently crafted titanium surfaces were very similar to those described for total log CFU. F. nucleatum was below the detection limit on all surfaces after $4 \mathrm{~h}$. Conclusion: Within the limitations of this in vitro study, surface roughness had a moderate influence on biofilm formation, while wettability did not seem to influence biofilm formation under the experimental conditions described. The modified SLA surface showed the highest trend for bacterial colonization.
\end{abstract}

Key words: Dental implants. Titanium. Biofilms. Surface properties. Wettability.

\section{INTRODUCTION}

The use of dental implants has become a routine procedure in dentistry to replace one or more missing teeth. Given implant survival as the main criteria for success, the majority of clinical studies show high success rates for dental implants ${ }^{4}$. However, there is also evidence of chronic inflammation, in the range of $8.6 \%-9.7 \%$, in soft and hard tissues neighboring implants ${ }^{12,15}$, and is commonly observed about ten years after implantation ${ }^{19}$. These pathologic conditions termed "mucositis" and "peri-implantitis", are considered major complications in dental implantology and their clinical manifestations such as gingival bleeding, swelling and bone loss, strongly resemble 
periodontal inflammation ${ }^{12}$. Numerous studies have shown a bacterial etiology with a similar spectrum of putative pathogens ${ }^{13}$ and a high concordance of bacterial species ${ }^{14,16}$. It is noteworthy that the colonization of "pristine" peri-implant pockets with periodontal disease associated bacteria has been shown to occur within 2 weeks ${ }^{17}$ and that some of these bacteria were found to be present as early as 30 min after insertion ${ }^{8}$. Biofilm formation on implant surfaces is therefore similar in composition and mechanisms known from teeth ${ }^{17}$, but may be additionally influenced by their special micro- and macroscopic design features.

Implants with smooth surfaces have been shown to exhibit a higher incidence of early failure, whereas implants with rougher surfaces display a lower incidence of early failure, but show increased failure rates over time ${ }^{8}$. Rough implant surfaces - while being beneficial for initial bone formation and osseointegration - have thus been generally considered to enhance initial adhesion and the subsequent colonization of oral bacteria ${ }^{18}$. Interactive energy effects, e.g. substratum hydrophobicity, surface-free energy and charge, however, must also be taken into consideration ${ }^{5}$.

This in vitro study assessed the extent of early biofilm colonization up to $16.5 \mathrm{~h}$ on titanium surfaces with different surface roughness and wetting characteristics. The hypothesis tested was that there would be no or only minute differences in the quantity and quality of biofilm formed over time. In this context, changes below 1 log step were considered to be irrelevant.

\section{MATERIAL AND METHODS}

\section{Disc preparation}

Round test specimens ( $5 \mathrm{~mm}$ diameter and $1 \mathrm{~mm}$ thickness) were manufactured from commercially pure (c.p.) grade 2 titanium (Straumann AG, $\mathrm{CH}-$ 4002, Basel, Switzerland) according to one of seven procedures, as described in Figure 1. Visual details of the microstructure of each surface are depicted in Figure 2. Modified surfaces (labeled "modSLA" and "modA") represent chemical modifications and were stored in glass ampoules containing the storage liquid, whereas all other samples were kept in air at room temperature. All specimens displayed different surface characteristics, i.e. surface roughness and wetting potentials, which were assessed as described below.

\section{Measurement of surface roughness and wetting ability}

Surface topography and roughness were analyzed by scanning electron microscopy (SEM) and white light confocal microscopy, respectively. Samples for SEM (Leo 1430, LEO Elektronenmikroskopie $\mathrm{GmbH}$, Oberkochen, Germany) were sputter coated (BAL-TEC SCD 050, BAL-TEC AG, Balzers, Liechtenstein) by a thin Au-Pd layer and examined at an accelerating voltage of $20 \mathrm{kV}$. The confocal three-dimensional (3D) white light microscope ( $\mu$ Surf, NanoFocus AG, Oberhausen, Germany) was used for surface topography measurements. An area of $798 \mu \mathrm{m} \times 770 \mu \mathrm{m}$ was measured to calculate the $3 \mathrm{D}$ roughness parameter Sa (arithmetic mean deviation of the surface) using a moving average Gaussian filter with a cut-off wavelength of 30 $\mu \mathrm{m}$. Five samples from each surface type were investigated.

Contact angle hysteresis was tensiometrically examined by the Wilhelmy method by means of an electrobalance (Sigma 70; KSV Instruments, Ltd., Helsinki, Finland). Dynamic contact angle analysis (DCA) was described in detail elsewhere 20,21 . The immersion velocity was set to $10 \mathrm{~mm} / \mathrm{min}$ for all experiments; the immersion depth was $15 \mathrm{~mm}$. All

\begin{tabular}{|c|l|}
\hline & \multicolumn{1}{|c|}{ Surface modification } \\
\hline Machined Sa>0.2 $\mathrm{mm}$ & Mechanically polished samples were prepared by using SiC gringing paper \\
\hline Machined Sa<0.2 $\mathrm{mm}$ & Mechanically polished samples were prepared by using SiC gringing paper \\
\hline $\mathrm{PT}$ & $\begin{array}{l}\text { Degreasing by washing in acetone, processing through } 2 \% \text { ammonium fluoride/2\% } \\
\text { hydrofluoric acid } / 10 \% \text { nitric acid solution at } 55^{\circ} \mathrm{C} \text { for } 30 \mathrm{~s}\end{array}$ \\
\hline $\mathrm{A}$ & $\begin{array}{l}\text { Acid-etching with a hot solution of } \mathrm{HCl} / \mathrm{H} 2 \mathrm{SO} 4 \text { according to a proprietary process of } \\
\text { Institut Straumann AG }\end{array}$ \\
\hline ModA & $\begin{array}{l}\text { New developed A surface. A surface will be rinsed under nitrogen protection and } \\
\text { then stored in a sealed glass tube containing isotonic NaCl solution at pH } 4 \text { to } 6\end{array}$ \\
\hline SLA & $\begin{array}{l}\text { Alumina blasting with a large grit particles (average particle size } 250 \mu \mathrm{m}) \text { and } \\
\text { subsequent acid-etching with a hot solution of } \mathrm{HCl} / \mathrm{H} 2 \mathrm{SO} 4 \text { according to a proprietary } \\
\text { process of Institut Straumann AG }\end{array}$ \\
\hline ModSLA & $\begin{array}{l}\text { Further development of SLA surface. SLA will be rinsed under nitrogen protection } \\
\text { and then stored in a sealed glass tube containing isotonic NaCl solution at pH } 4 \text { to } 6\end{array}$ \\
\hline
\end{tabular}

Figure 1-Surfaces and surface modifications investigated in this study 
multi-loop experiments have been repeated at least fourfold at room temperature. Five samples from each surface type were investigated.

\section{Biofilm preparation}

The experiments were repeated 3 times in triplicates for each surface, resulting in a sample size of 9 specimens per group.

Actinomyces oris OMZ 745, Veillonella dispar ATCC 17748T (OMZ 493), Fusobacterium nucleatum KP-F2 (OMZ 596), Streptococcus sobrinus OMZ 176, Streptococcus oralis SK248 (OMZ 607), and Candida albicans OMZ 110 were used as inocula for biofilm formation ${ }^{10,22,24}$. In brief, all microorganisms were grown to the mid logarithmic phase using a strictly controlled incubation period for each species. In numerous preliminary experiments, growth curves of all microorganisms using culture techniques and optical density measurements were made. Thus, the incubation time of each strain of consortium to reach the mid logarithmic phase could be determined. The density of aliquots of the cultures was measured and adjusted to an absorption of $1.0(\lambda 550 \mathrm{~nm})$. The density of the cultures was adjusted accordingly and $1 \mathrm{~mL}$ of each culture was used to prepare the final inoculum ${ }^{10}$. The inoculum contained reproducibly $10^{7}-10^{8}$ microorganisms of each species per ml. Biofilms were grown in 24-well polystyrene cell culture plates ${ }^{25}$. For this purpose, discs were preconditioned (pellicle-coated) in processed whole unstimulated pooled human saliva and were cultured as shown in Figure 3. The collection of saliva and the preconditioning of discs have been previously described ${ }^{10}$. Whole, un-stimulated saliva was obtained over several days from volunteers (with informed consent) at least $1.5 \mathrm{~h}$ after eating, drinking, or tooth cleaning. Saliva samples, collected in sterile $50 \mathrm{~mL}$ polypropylene tubes chilled in an ice bath, were frozen at $-20^{\circ} \mathrm{C}$. When a total of ca. $500 \mathrm{~mL}$ saliva had been collected, it was pooled and centrifuged (30 $\mathrm{min}, 4^{\circ} \mathrm{C}, 27,000 \mathrm{xg}$ ), and the supernatant was pasteurized $\left(60^{\circ} \mathrm{C}, 30 \mathrm{~min}\right)$ and re-centrifuged in sterile bottles; the resulting supernatant was dispensed into sterile $50-\mathrm{mL}$ polypropylene tubes and stored at $20^{\circ} \mathrm{C}$. The efficacy of pasteurization was assessed by plating processed saliva samples onto CBA; after $72 \mathrm{~h}$ at $37^{\circ} \mathrm{C}$, no colony forming units (CFU) were observed on either aerobically or anaerobically incubated plates.

To allow formation of a salivary pellicle, titanium disc were incubated in sterile 24-well polystyrene cell culture plates (NuncA/S, Roskilde, Denmark), with processed saliva (for $4 \mathrm{~h}$, gently shaken, at room temperature). Saliva was aspirated from each well and replaced with $800 \mathrm{~mL}$ saliva and 800 $\mathrm{mL} \mathrm{mFUM}$ medium containing $0.15 \%$ glucose and $0.15 \%$ sucrose. The wells were inoculated with the

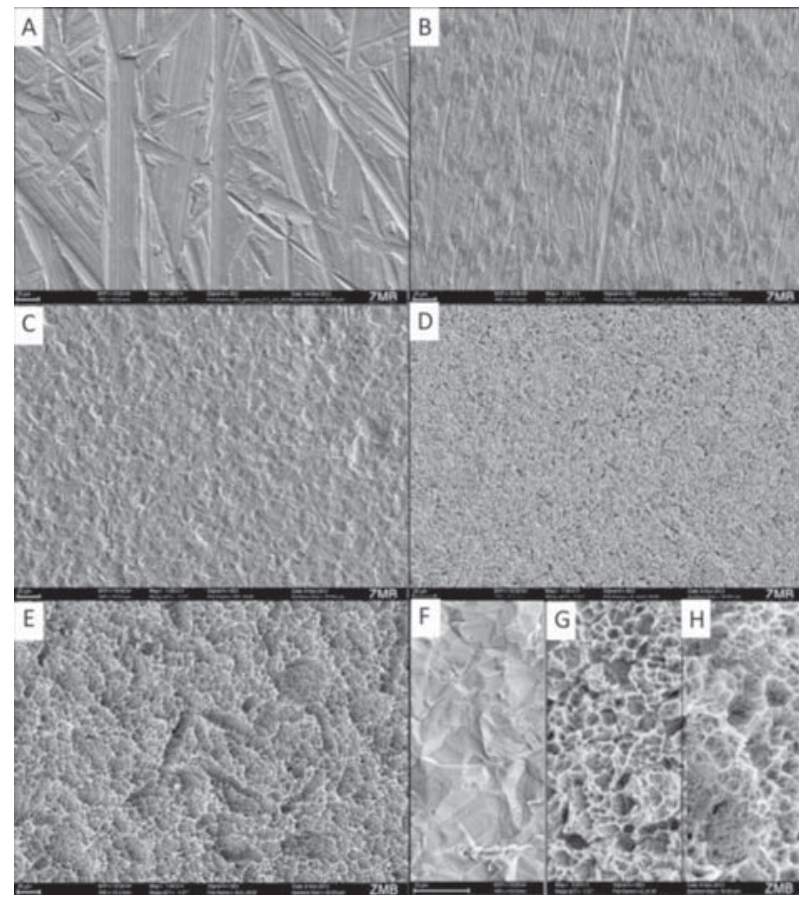

Figure 2- Scanning electron microscopy images of the different titanium surfaces at magnifications of $500 x(A-E)$ and 1,000x (E-G), respectively. A: machined Sa>0.2 $\mu \mathrm{m}$; B: machined $\mathrm{Sa}<0.2 \mu \mathrm{m}$; C: stained; D: acid-etched; E: sandblasted/acid-etched (SLA). Modified surfaces did not differ in their microscopic surface characteristics. Details at higher magnification of images $\mathrm{C}-\mathrm{E}$ are given in $\mathrm{F}$ : stained PT; G: acid-etched and H: SLA

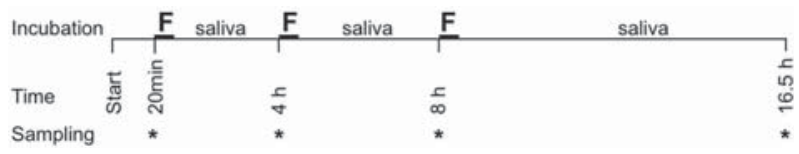

Figure 3- Schematic timeline of the experimental conditions ( $F$ represents $45 \mathrm{~min}$ feeding periods with saliva/mFUM 30/70). Between feedings, the discs were incubated in saliva

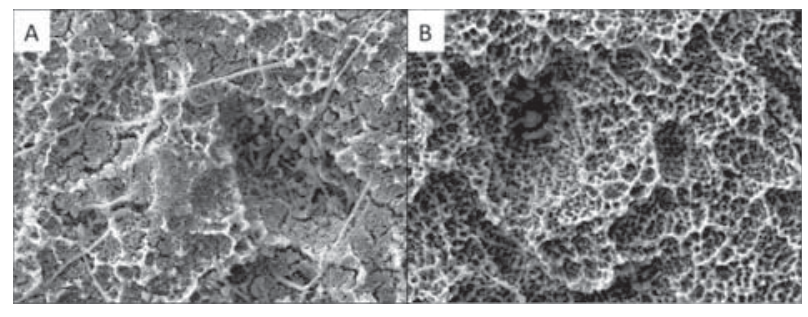

Figure 4- Scanning electron microscophy images of sandblasted/acid-etched (SLA) surfaces (before treatment see figure $2 \mathrm{E}$ and $\mathrm{H}$ ) after $16.5 \mathrm{~h}$ biofilm formation before $(A)$ and after vortexing $(B)$. Coherent mats of microorganisms cover the surface (A). Only few bacteria remain on the surface after harvest by vortexing (B). The surface is comparable to the original surface (Figure $2 \mathrm{E} / \mathrm{H}$ ) 
pooled oral microbiota $(200 \mathrm{~mL})$ for $20 \mathrm{~min}$.

Following an initial adherence period of $20 \mathrm{~min}$, the biofilms were fed with the same medium for $45 \mathrm{~min}$ and again after 4 and $8 \mathrm{~h}$ incubation. After each feeding, the discs were $3 x$ dip-washed in saline to remove non-adhering microorganisms. During the remaining time, the biofilms were incubated in saliva. Biofilms were harvested after $20 \mathrm{~min}, 2,8$ and $16.5 \mathrm{~h}$ (Figure 4).

\section{Harvesting and examination of the biofilm microbiota}

To harvest adherent microorganisms, each disc was transferred to a sterile $50 \mathrm{ml}$ polypropylene tube containing physiological saline $(1 \mathrm{~mL}$, room temperature) and vortexed vigorously for $2 \mathrm{~min}$. The suspensions were then transferred to sterile 6-ml polystyrene tubes and sonified for $5 \mathrm{~s}$ at 30 W. Serial dilutions $\left(10^{-2}-10^{-5}\right)$ of sonified cells were prepared in physiological saline and aliquots (50 $\mathrm{mL}$ ) were spirally plated (Spiral System, Model D, Spiral Systems, Inc., Cincinnati, USA) onto CBA plates (Columbia Blood Agar, Oxoid, CM $331+5 \%$ whole human blood) for assessing total CFU and as well for identifying and counting A.naes/undi and V.dispar. F.nucleatum was counted using an FAA [Fastidious Anaerobe Agar (lab m, UK, BAG, 7621)] supplemented with erythromycin ( $1 \mathrm{mg} / \mathrm{L})$ Sigma, E-6376/dissolved in distilled $\mathrm{H}_{2} \mathrm{O}$, vancomycin (4 mg/L) Lilly, 657/dissolved in distilled $\mathrm{H}_{2} \mathrm{O}$ and norfloxacin (1 mg/L) Sigma, N-9890/dissolved in absolute ethanol. S. sobrinus and $S$. oralis colonies were assessed on MS agar (Mitis-Salivarius agar, Difco 0298-17-2, Heidelberg, Germany) and $C$. albicans on Biggy-Agar (Difco 0635-17-4). After 72 $h$ incubation, CFUs were counted with the assistance of a stereomicroscope.

Data presentation and statistical analysis

Statistical analysis was performed with a commercially available program (StatView, Version 5, Abacus Concepts Inc., Berkeley, USA). For the determination of the surface roughness $\left(S_{a}, \mu \mathrm{m}\right)$ and contact angle $\left(q,{ }^{\circ}\right)$ measurements, mean values and standard deviations were calculated.

For the biofilm evaluation, mean values and corresponding 95\% confidence intervals (95\% $\mathrm{CI})$ were calculated. To determine the differences

Table 1- Arithmetic mean deviation of the surface $\left(S_{a}, \mu m\right)$ types and respective contact angle values (q, $\left.{ }^{\circ}\right)$. SLA: Sandblasted/acid-etched

\begin{tabular}{cccccccc}
\hline $\begin{array}{c}\text { Roughness } \\
\text { Parameter }\end{array}$ & $\begin{array}{c}\text { Machined } \\
\mathrm{S}_{\mathrm{a}}>\mathbf{0 . 2} \boldsymbol{\mu m}\end{array}$ & $\begin{array}{c}\text { Machined } \\
\mathrm{S}_{\mathrm{a}}>\mathbf{0 . 2} \boldsymbol{\mu m}\end{array}$ & Stained & Acid-etched & $\begin{array}{c}\text { Modified Acid- } \\
\text { etched }\end{array}$ & $\begin{array}{c}\text { SLA } \\
\text { Modified } \\
\text { SLA }\end{array}$ \\
\hline $\mathrm{Sa}$ & $0.3 \pm 0.06$ & $0.1 \pm 0.02$ & $0.4 \pm 0.07$ & $0.6 \pm 0.01$ & $0.6 \pm 0.02$ & $1.3 \pm 0.06$ & $1.2 \pm 0.09$ \\
$\mathrm{q}\left({ }^{\circ}\right)$ & $92 \pm 5$ & $92 \pm 5$ & $93 \pm 5$ & $121 \pm 4$ & 0 & $134 \pm 5$ & 0 \\
\hline
\end{tabular}

Table 2- Total log colony forming units (CFU) (mean values and $95 \%$ confidence intervals in parentheses; $N=9$ per group) on the various surfaces at the given time points

\begin{tabular}{|c|c|c|c|c|}
\hline & $20 \mathrm{~min}$ & $4 h$ & $8 \mathrm{~h}$ & $16.5 \mathrm{~h}$ \\
\hline Machined & $4.5^{\mathrm{A}}$ & $3.9^{A}$ & $4.8^{\mathrm{AB}}$ & $5.9^{\mathrm{ABCD}}$ \\
\hline $\mathrm{S}_{\mathrm{a}}<0.2 \mu \mathrm{m}$ & $(4.3,4.7)$ & $(3.6,4.2)$ & $(4.6,5.0)$ & $(5.6,6.3)$ \\
\hline \multirow[t]{2}{*}{ Stained } & $4.3^{\mathrm{A}}$ & $3.9^{A}$ & $4.6^{A}$ & $5.4^{\mathrm{AB}}$ \\
\hline & $(4.1,4.5)$ & $(3.7,4.1)$ & $(4.4,4.7)$ & $(5.2,5.7)$ \\
\hline \multirow[t]{2}{*}{ Machined $\mathrm{Sa}>0.2 \mu \mathrm{m}$} & $4.5^{\mathrm{A}}$ & $3.7^{\mathrm{A}}$ & $4.9^{A B}$ & $5.9 \mathrm{ABCD}$ \\
\hline & $(4.4,4.7)$ & $(3.4,4.0)$ & $(4.6,5.2)$ & $(5.7,6.2)$ \\
\hline \multirow[t]{2}{*}{ Acid-etched } & $4.5 \mathrm{~A}$ & $3.8^{A}$ & $4.9^{A B}$ & $5.7^{\mathrm{BD}}$ \\
\hline & $(4.4,4.7)$ & $(3.5,4.2)$ & $(4.7,5.4)$ & $(5.3,6.2)$ \\
\hline \multirow[t]{2}{*}{ Modified acid-etched } & $4.5^{\mathrm{A}}$ & $4.3^{\mathrm{A}}$ & $5.4^{\mathrm{BC}}$ & $6.5^{\mathrm{CE}}$ \\
\hline & $(4.3,4.7)$ & $(4.0,4.5)$ & $(5.3,5.4)$ & $(6.4,6.6)$ \\
\hline \multirow[t]{2}{*}{ SLA } & $4.7^{A B}$ & $4.2^{\mathrm{A}}$ & $5.6^{c}$ & $6.5^{\mathrm{DE}}$ \\
\hline & $(4.6,4.8)$ & $(3.9,4.5)$ & $(5.4,5.8)$ & $(6.3,6.6)$ \\
\hline \multirow[t]{2}{*}{ Modified SLA } & $4.8^{\mathrm{B}}$ & $4.8^{\mathrm{B}}$ & $6.2^{\mathrm{D}}$ & $6.9 \mathrm{E}$ \\
\hline & $(4.8,4.9)$ & $(4.7,4.9)$ & $(5.9,6.5)$ & $(6.7,7.0)$ \\
\hline
\end{tabular}

Different superscript letters represent significant differences (ANOVA and Scheffé post hoc analysis, read vertically). SLA: Sandblasted/acid-etched 
between smooth and rough surfaces at respective biofilm formation times, an unpaired t-test was used. One-way analysis of variance (ANOVA), together with the post hoc Scheffé test, was applied to establish the differences between the different materials. The significance level was set at $95 \%$.

Table 3- Colony forming units (log CFU, mean values and 95\% confidence intervals in parentheses) of the different species determined on the various surfaces at the given time points ( $\mathrm{N}=9$ per group; ANOVA and Scheffé post hoc analysis)

\begin{tabular}{|c|c|c|c|c|c|}
\hline & & $20 \mathrm{~min}$ & $4 h$ & $8 \mathrm{~h}$ & $16.5 \mathrm{~h}$ \\
\hline \multirow[t]{7}{*}{ A. naeslundii } & $\mathrm{S}_{\mathrm{a}}<0.2 \mu \mathrm{m}$ & $3.1(2.8 ; 3.4)$ & $1.3(1.2 ; 1.5)$ & $2.5(2.2 ; 2.8)$ & $2.3(2.3 ; 2.4)$ \\
\hline & Stained & $3.1(2.9 ; 3.2)$ & $2.0(1.4 ; 2.7)$ & $2.2(1.8 ; 2.7)$ & $2.4(2.2 ; 2.6)$ \\
\hline & $S_{a}>0.2 \mu m$ & $2.9(2.8 ; 3.0)$ & - & $2.5(2.3 ; 2.7)$ & $2.4(2.2 ; 2.7)$ \\
\hline & acid-etched & $3.3(3.2 ; 3.5)$ & $1.5(1.3 ; 1.8)$ & $2.2(1.6 ; 2.8)$ & $2.4(2.2 ; 2.7)$ \\
\hline & $\begin{array}{l}\text { mod. acid-etched } \\
\text { SLA }\end{array}$ & $3.4(3.3 ; 3.6)$ & $1.8(1.3 ; 2.3)$ & $2.8(2.4 ; 3.2)$ & $3.4(3.3 ; 3.6)$ \\
\hline & SLA & $3.7(3.6 ; 3.9)$ & $1.7(1.4 ; 2.0)$ & $3.2(2.9 ; 3.6)$ & $3.6(3.2 ; 3.9)$ \\
\hline & mod. SLA & $3.9(3.7 ; 4.0)$ & $2.1(1.7 ; 2.6)$ & $3.7(3.5 ; 4.0)$ & $4.2(3.7 ; 4.7)$ \\
\hline \multirow[t]{7}{*}{ V. dispar } & $\mathrm{S}_{\mathrm{a}}<0.2 \mu \mathrm{m}$ & $4.4(4.2 ; 4.6)$ & $3.8(3.5 ; 4.1)$ & $4.3(4.2 ; 4.5)$ & $5.9(5.6 ; 6.3)$ \\
\hline & Stained & $4.2(4.0 ; 4.4)$ & $3.8(3.7 ; 4.0)$ & $4.1(4.0 ; 4.3)$ & $5.4(5.1 ; 5.6)$ \\
\hline & $\mathrm{Sa}>0.2 \mu \mathrm{m}$ & $4.2(3.9 ; 4.6)$ & $3.5(3.1 ; 4.0)$ & $4.6(4.3 ; 4.9)$ & $5.9(5.6 ; 6.2)$ \\
\hline & acid-etched & $4.4(4.2 ; 4.6)$ & $3.7(3.4 ; 4.0)$ & $4.4(4.1 ; 4.7)$ & $5.7(5.2 ; 6.1)$ \\
\hline & $\begin{array}{l}\text { mod. acid-etched } \\
\text { SLA }\end{array}$ & $4.5(4.2 ; 4.7)$ & $4.2(3.9 ; 4.4)$ & $4.9(4.7 ; 5.2)$ & $6.5(6.3 ; 6.6)$ \\
\hline & SLA & $4.5(4.3 ; 4.7)$ & $4.0(3.8 ; 4.3)$ & $5.3(5.1 ; 5.5)$ & $6.5(6.3 ; 6.6)$ \\
\hline & mod. SLA & $4.6(4.6 ; 4.7)$ & $4.7(4.5 ; 4.8)$ & $5.9(5.7 ; 6.1)$ & $6.8(6.5 ; 7.0)$ \\
\hline \multirow[t]{7}{*}{ F. nucleatum } & $S_{a}<0.2 \mu m$ & $1.4(1.2 ; 1.6)$ & - & - & - \\
\hline & Stained & $1.4(1.3 ; 1.5)$ & - & - & - \\
\hline & $S_{a}>0.2 \mu \mathrm{m}$ & - & - & - & - \\
\hline & acid-etched & $1.7(1.2 ; 2.1)$ & - & - & - \\
\hline & $\begin{array}{l}\text { mod. acid-etched } \\
\text { SLA }\end{array}$ & $1.5(1.3 ; 1.7)$ & - & - & - \\
\hline & SLA & $1.5(1.2 ; 1.7)$ & $1.5(1.3 ; 1.7)$ & - & - \\
\hline & mod. SLA & $1.7(1.2 ; 2.1)$ & - & - & - \\
\hline \multirow[t]{7}{*}{ S. sobrinus } & $\mathrm{S}_{\mathrm{a}}<0.2 \mu \mathrm{m}$ & $2.1(1.6 ; 2.5)$ & $2.3(2.0 ; 2.7)$ & $4.3(3.8 ; 4.8)$ & $4.4(4.1 ; 4.7)$ \\
\hline & Stained & $1.6(1.4 ; 1.8)$ & $2.2(1.6 ; 2.7)$ & $4.0(3.7 ; 4.4)$ & $4.0(3.8 ; 4.2)$ \\
\hline & $S_{a}>0.2 \mu \mathrm{m}$ & $1.7(1.5 ; 2.0)$ & $2.1(1.8 ; 2.4)$ & $4.3(3.7 ; 4.9)$ & $4.3(4.1 ; 4.5)$ \\
\hline & acid-etched & $1.9(1.6 ; 2.2)$ & $2.6(2.0 ; 3.2)$ & $4.3(3.4 ; 5.3)$ & $4.5(4.0 ; 4.9)$ \\
\hline & $\begin{array}{l}\text { mod. acid-etched } \\
\text { SLA }\end{array}$ & $2.0(1.6 ; 2.3)$ & $3.1(2.6 ; 3.6)$ & $4.6(4.0 ; 5.1)$ & $4.8(4.5 ; 5.1)$ \\
\hline & SLA & $2.2(1.9 ; 2.5)$ & $3.1(2.5 ; 3.7)$ & $5.0(4.4 ; 5.6)$ & $5.1(4.8 ; 5.4)$ \\
\hline & mod. SLA & $2.6(2.3 ; 2.9)$ & $3.5(2.8 ; 4.2)$ & $5.5(5.0 ; 5.9)$ & $5.6(5.4 ; 5.9)$ \\
\hline \multirow[t]{7}{*}{ S. oralis } & $\mathrm{S}_{\mathrm{a}}<0.2 \mu \mathrm{m}$ & $\begin{array}{c}2.0(1.8 ; 2.3) 2.1 \\
(1.9 ; 2.3)\end{array}$ & $2.1(1.9 ; 2.3)$ & $2.9(2.6 ; 3.2)$ & $3.9(3.6 ; 4.2)$ \\
\hline & Stained & $2.0(1.7 ; 2.3)$ & $2.1(1.6 ; 2.5)$ & $2.6(2.2 ; 3.0)$ & $3.4(3.0 ; 3.7)$ \\
\hline & $S_{a}>0.2 \mu \mathrm{m}$ & $2.1(1.9 ; 2.3)$ & $1.9(1.5 ; 2.2)$ & $3.1(2.7 ; 3.4)$ & $3.6(3.3 ; 3.8)$ \\
\hline & acid-etched & $2.0(1.8 ; 2.6)$ & $2.6(2.3 ; 2.8)$ & $3.3(2.9 ; 3.6)$ & $3.7(3.4 ; 4.0)$ \\
\hline & $\begin{array}{c}\text { mod. acid-etched } \\
\text { SLA }\end{array}$ & $2.6(2.0 ; 3.2)$ & $2.5(2.2 ; 2.9)$ & $3.5(3.1 ; 3.8)$ & $4.0(3.8 ; 4.2)$ \\
\hline & SLA & $2.4(2.2 ; 2.5)$ & $2.6(2.1 ; 3.0)$ & $3.7(3.4 ; 4.0)$ & $4.2(4.0 ; 4.4)$ \\
\hline & mod. SLA & & $2.5(2.2 ; 2.9)$ & $4.2(4.0 ; 4.4)$ & $4.7(4.4 ; 5.1)$ \\
\hline \multirow[t]{7}{*}{ C. albicans } & $S_{a}<0.2 \mu m$ & $3.0(2.6 ; 3.3)$ & $1.8(1.6 ; 2.1)$ & $2.4(2.0 ; 2.7)$ & $2.3(2.1 ; 2.5)$ \\
\hline & Stained & $3.1(2.9 ; 3.2)$ & $2.4(2.0 ; 2.8)$ & $2.2(2.0 ; 2.4)$ & $2.1(1.8 ; 2.4)$ \\
\hline & $S_{a}>0.2 \mu \mathrm{m}$ & $3.2(3.0 ; 3.4)$ & $2.0(1.7 ; 2.4)$ & $2.7(2.4 ; 2.9)$ & $2.1(1.7 ; 2.4)$ \\
\hline & acid-etched & $3.6(3.3 ; 3.9)$ & $2.0(1.6 ; 2.5)$ & $2.0(1.6 ; 2.4)$ & $2.0(1.7 ; 2.3)$ \\
\hline & $\begin{array}{l}\text { mod. acid-etched } \\
\text { SLA }\end{array}$ & $3.2(3.1 ; 3.4)$ & $2.3(1.8 ; 2.9)$ & $2.3(1.9 ; 2.8)$ & $2.4(2.1 ; 2.7)$ \\
\hline & SLA & $3.9(3.8 ; 4.0)$ & $3.1(2.7 ; 3.5)$ & $3.5(3.1 ; 3.9)$ & $3.0(2.8 ; 3.1)$ \\
\hline & mod. SLA & $3.9(3.8 ; 4.0)$ & $3.6(3.4 ; 3.9)$ & $3.7(3.5 ; 4.0)$ & $3.2(3.2 ; 3.3)$ \\
\hline
\end{tabular}




\section{RESULTS}

\section{Surface roughness and wetting ability}

The measured values are presented and summarized in Table 1.

$S_{a}$ values $>1 \mu \mathrm{m}$ were only obtained for the SLA surfaces. The acid-etched surfaces had mean $\mathrm{S}_{\mathrm{a}}$ values of $0.6 \mu \mathrm{m}$. The chemically modified and activated surfaces showed no statistically significant difference as compared to their un-activated counterparts. All other samples showed a mean surface roughness below $0.5 \mu \mathrm{m}$, with the more polished surface reaching mean $\mathrm{S}_{\mathrm{a}}$ values of $0.1 \mu \mathrm{m}$.

With regard to the contact angle measurements, it can be summarize that the best wetting ability was achieved by the modified (i.e. activated) samples $(q=10)$. The $q$-values of the acid-etched and SLA surfaces were the highest, with values of 121 and 134, respectively. This indicates very low surface wetting characteristics. The results of the polished and stained surfaces ranged between $92^{\circ}$ and $93^{\circ}$.

\section{Biofilm formation}

The results of the microbial biofilm analysis are summarized in Tables 2 and 3.

Figure 3A shows a SLA surface after incubation. A strong microbial colonization was evident after $16.5 \mathrm{~h}$ of incubation.

The mean number of microorganisms adhering to the discs after 20 min showed only minor differences between the groups and ranged between 4.5 to $4.8 \log _{10}$ CFU. The highest initial adherence was observed on modified SLA discs $(P \leq 0.01)$.

After the first feeding period (4 $\mathrm{h}$ of incubation), there was no growth on all surfaces observed. In contrast, all test groups showed a colonization decrease of 0.2 to 0.7 log steps, with the exception of the modified SLA, as compared to the initial adherence phase.

After $8 \mathrm{~h}$, the particular pattern of microbial colonization on the different titanium surfaces persisted, however, at this time point there was significantly increased growth observed on all test surfaces. In the following time period up to $16.5 \mathrm{~h}$, growth continued further with a low but material specific rate (Table 2 ).

Regarding the number of initially adhering microorganisms (Table 3), $v$. dispar showed by far the strongest adherence to these titanium surfaces ( $\approx \log 4$ steps). S. oralis and C. albicans exhibited a one log step lower adherence.

After $4 \mathrm{~h}$ incubation, the microbial composition of the biofilms had started to shift. $V$. dispar remained at the level of initial adherence. The numbers of $S$. oralis and C. albicans decreased dramatically while S. sobrinus, started to increase. F. nucleatum fell, with one exception (SLA), below detection level.
After $8 \mathrm{~h}$ incubation, biofilm growth increased again: $V$. dispar increased by approx. 1 log step reaching a level of $\log 5 ; S$. sobrinus continued to grow, almost attaining the level of $V$. dispar; and $S$. oralis continued to grow, albeit at a lower growth rate. The colonization density of $C$. albicans remained unchanged.

From the $8 \mathrm{~h}$ reading to the end of the experiment at $16.5 \mathrm{~h}$, microbial density continued to increase, but at a slower rate. It was evident that $V$. dispar was primarily responsible for this increase, and seems to have profited from the lactic acid produced by the streptococci as an energy source. Both streptococcal species only showed minute changes. The other species remained stagnant. $F$. nucleatum colonized during the entire experimental period near or below detection level.

\section{DISCUSSION}

The results of this study support the hypothesis that regardless of the titanium's surface roughness and despite highly significant differences in wetting properties, bacterial colonization was quite similar on all implant materials over time. The differences between the different colonization mass was within the range of one log step. That rough and smooth machined surface values showed quite comparable colonization in this study is in contrast to the existing opinion of a threshold value of $0.2 \mu \mathrm{m}^{23}$.

The so-called "Zurich biofilm model" was applied, which has been validated in several studies ${ }^{10,11}$. Although this model represents a supragingival plaque model, its relative ease to cultivate, as well as its basic biofilm characteristics and role in the ensuing development of subgingival plaque/periimplantitis, allow for a realistic and reproducible laboratory simulation of the oral condition.

With the exception of $C$. albicans, all selected bacteria are found in high numbers in supra-gingival plaque responsible for gingivitis. Colonization of implants preceding pocket formation is dependent on initial inflammation occurring in the absence of sufficient dental hygiene measures. The subgingival microbiota responsible for peri-implantitis, including in addition high numbers of anaerobic Gramnegative bacteria, can only establish gradually after inflammation and pocket formation have progressed. As Gram-positive bacteria form the first layer of firmly adherent microorganisms on the root surface, selection of the present biofilm consortium cannot be considered too far-fetched ${ }^{26}$.

The spatial arrangement and the associative behavior are well documented and the model has been used to assess different aspects in microbiology as well as clinically based and oriented research in the dental field ${ }^{10}$. Whereas the experiments described above were predominantly 
performed on sterile hydroxyapatite discs, this is the first study assessing the biofilm growth on titanium samples. In another laboratory study, the applicability of our model was assessed on other dental materials ${ }^{6}$. Similar to the present study, the influence of surface roughness and contact time on the formation of a multi-species biofilm on different materials was tested. This earlier study showed that surface roughness may influence initial biofilm adherence after 15 min, but differences vanished following growth and maturation phases after $15 \mathrm{~h}$, irrespective of whether rough or smooth surfaces were tested.

Within the limitations of the employed study design, the results are in line with implants of different surface characteristics, although moderately rough surfaces (Sa between 1 and 2 $\mu \mathrm{m}$ ) tended towards higher plaque accumulation ${ }^{1}$. A recently published in situ study revealed a significantly lower biofilm adherence after $2 \mathrm{~h}$ on smooth turned titanium surfaces, however, after 14 $h$, the biofilm volume on all surfaces was similar again, suggesting that the influence of surface characteristics on adhesion was compensated for by biofilm development, which is again in line with our findings?. Another in vitro evaluation used the same implant surface types and a slightly modified Zurich biofilm model incorporating 9 bacterial strains, which were assessed after a $24 \mathrm{~h}$ incubation using either culture medium or human saliva, with checkerboard analysis ${ }^{2}$. The saliva incubation technique corresponds to our set-up and showed lower counts of bacteria as compared to culture medium. This study also showed that SLA and modified SLA had a significant increase in bacterial adhesion when using human saliva. The findings of the study showed by micro-topography that biofilm formation and composition was affected by hydrophilicity of the surface. This observation is not supported by our results. This difference may be explained, only in part, by the different bacterial strains used but not by the microbiological evaluation techniques (culture versus checkerboard analysis).

The investigation of the initial adherence and growth of the 6 microbial species on these differently crafted titanium surfaces provided other interesting insights. The strongest initial adherence among the 6 microorganisms used for biofilm formation was exhibited by $V$. dispar and $C$. albicans. Among the differently crafted titanium surfaces, adherence differences were very similar to those described for total CFU. Differences in affinity of $A$. oris for these surfaces were more distinct. The lowest adherence was observed on stained titanium and on surfaces with a roughness $<0.2 \mu \mathrm{m}$. A higher but still low affinity for this species was observed on the acid etched, modified acid etched and titanium surfaces with a roughness of $>0.2 \mu \mathrm{m}$. Significantly stronger adherence, in comparison with the acid etched and modified etched surfaces, were evident for the SLA $(P f<0.5)$ and even more for the modified SLA surfaces $(\mathrm{Pf}<0.01)$. F. nucleatum remained, with one exception, below detection level. That is not surprising. In all biofilm experiments within the last ten years, this particular bacterial strain hibernated during the first $16.5 \mathrm{~h}$ and only started to grow on saliva-preconditioned hydroxyapatite disks in the period between $16.5 \mathrm{~h}$ and $64.5 \mathrm{~h}$. It appears that the accompanying microbiota has to first prime the conditions in the biofilm, to allow growth of this fastidious species. The proportion of $F$. nucleatum in a study by Almaguer-Flores and co-workers was also low after $24 \mathrm{~h}$, which supports our observation ${ }^{2}$. Astonishingly low was also the adherence of the streptococci on all titanium surfaces. This is in contrast to biofilm experiments with natural tooth or hydroxyapatite surfaces. Guggenheim and co-workers showed an initial adherence for $S$. mutans and $S$. oralis in the order of magnitude of log 4 on pellicle-coated hydroxyapatite discs of similar size ${ }^{10}$. The influence of structural, physical and chemical micro-surface characteristics should not be neglected, as it has been demonstrated in some studies that these factors, including the microenvironment, may influence the attachment and growth of individual microorganisms ${ }^{2,3,9}$.

After the first feeding period and during the 4 $h$ of incubation, detachment of all microorganisms was observed. The intensity of this shedding was primarily dependent upon the nature of the titanium surface structure and showed substantial differences between species. Consistent at this time point was the highly significant difference $(P f<0.01)$ between the modified SLA surface, showing the highest number of microorganisms, and the titanium surfaces with a surface roughness $<$ and $>0.2$. However, after $8 \mathrm{~h}$, all species on all surfaces showed re-growth, with the exception of C. albicans and $S$. sobrinus on the titanium surfaces with surface roughness $<0.2$ and $>0.2 \mu \mathrm{m}$. Up to $16.5 \mathrm{~h}$ growth continued and the colonization level of $A$. oris and $V$. dispar exceeded in numbers the initial adherence counts. Both streptococcal species showed growth reaching the density of the initial adherence phase while the numbers of $C$. albicans remained almost unchanged. This failure of $C$. albicans to grow, however, may be explained by the anaerobic incubation of the biofilms.

In summary, it was found that titanium disks with moderately rough surfaces tended to exhibit more biofilm formation patterns. However, most differences in colonization density between the tested titanium surfaces did not reach statistical significance. Biofilm formation on implant surfaces appeared to be controlled not only by growth 
conditions, but also, albeit to a minor extent, by the nature of the colonized surface. Although the adherence pattern of the total biofilm microbiota on the different titanium surfaces remained remarkably constant through out the experiment, the minute differences visible after the initial adherence phase became more prominent over time but ranged within one log step. The surface modification had no effect on biofilm formation.

\section{CONCLUSION}

Surface roughness moderately influenced biofilm formation under the experimental conditions described, whereas wettability was less influential. From a clinical point of view, the implant surface needs daily meticulous oral hygiene. If this condition is met, then the choice of implant surface characteristics may be salient to the long-term health of any implant placed. However, if the biofilm is allowed to grow uncontrolled, the influences of different titanium surfaces become irrelevant.

\section{REFERENCES}

1- Albrektsson T, Wennerberg A. Oral implant surfaces: Part 1 - review focusing on topographic and chemical properties of different surfaces and in vivo responses to them. Int J Prosthodont. 2004;17:536-43.

2- Almaguer-Flores $A$, Olivares-Navarrete $R$, Wieland $M$, Ximénez-Fyvie LA, Schwartz $Z$, Boyan BD. Influence of topography and hydrophilicity on initial oral biofilm formation on microstructured titanium surfaces in vitro. Clin Oral Implants Res. 2012;23:301-7.

3- Amoroso PF, Adams RJ, Waters MG, Williams DW. Titanium surface modification and its effect on the adherence of Porphyromonas gingivalis: an in vitro study. Clin Oral Implants Res. 2006;17:633-7.

4- Blanes RJ, Bernard JP, Blanes ZM, Belser UC. A 10-year prospective study of ITI dental implants placed in the posterior region. II: Influence of the crown-to-implant ratio and different prosthetic treatment modalities on crestal bone loss. Clin Oral Implants Res. 2007;18:707-14. 5- Busscher HJ, Rinastiti M, Siswomihardjo W, van der Mei HC. Biofilm formation on dental restorative and implant materials. J Dent Res. 2010;89:657-65.

6- Dezelic T, Guggenheim B, Schmidlin PR. Multi-species biofilm formation on dental materials and an adhesive patch. Oral Health Prev Dent. 2009;7:47-53.

7- Fröjd V, Chávez de Paz L, Andersson M, Wennerberg A, Davies JR, Svensater G. In situ analysis of multispecies biofilm formation on customized titanium surfaces. Mol Oral Microbiol. 2011;26:241-52.

8- Furst MM, Salvi GE, Lang NP, Persson GR. Bacterial colonization immediately after installation on oral titanium implants. Clin Oral Implants Res. 2007;18:501-8.

9- Groessner-Schreiber B, Hannig M, Dück A, Griepentrog M, Wenderoth DF. Do different implant surfaces exposed in the oral cavity of humans show different biofilm compositions and activities? Eur ] Oral Sci. 2004;112:51622.
10- Guggenheim B, Giertsen E, Schüpbach P, Shapiro S. Validation of an in vitro biofilm model of supragingival plaque. J Dent Res. 2001;80:363-70.

11- Guggenheim B, Guggenheim M, Gmür R, Giertsen E, Thurnheer T. Application of the Zurich biofilm model to problems of cariology. Caries Res. 2004;38:212-22.

12- Jung RE, Pjetursson BE, Glauser R, Zembic A, Zwahlen $M$, Lang NP. A systematic review of the 5 -year survival and complication rates of implant-supported single crowns. Clin Oral Implants Res. 2008;19:119-30.

13- Meffert RM. Periodontitis vs. peri-implantitis: the same disease? The same treatment? Crit Rev Oral Biol Med. 1996;7:278-91.

14- Mombelli A. Microbiology of the dental implant. Adv Dent Res. 1993; 7:202-6.

15- Pjetursson BE, Tan K, Lang NP, Brägger U, Egger M, Zwahlen M. A systematic review of the survival and complication rates of fixed partial dentures (FPDs) after an observation period of at least 5 years. Clin Oral Implants Res. 2004;15:667-76.

16- Pontoriero R, Tonelli MP, Carnevale G, Mombelli A, Nyman SR, Lang NP. Experimentally induced peri-implant mucositis. A clinical study in humans. Clin Oral Implants Res. 1994;5:254-9.

17- Quirynen $M$, van der Mei HC, Bollen $C M$, van den Bossche LH, Doornbusch GI, van Steenberghe D. The influence of surface-free energy on supra- and subgingival plaque microbiology. An in vivo study on implants. J Periodontol. 1994;65:162-7.

18- Quirynen M, Vogels R, Peeters W, van Steenberghe $D$, Naert I, Haffajee A. Dynamics of initial subgingival colonization of "pristine" peri-implant pockets. Clin Oral Implants Res. 2006;17:25-37.

19- Roos-Jansåker AM, Renvert $\mathrm{H}$, Lindahl C, Renvert S. Nine- to fourteen-year follow-up of implant treatment. Part III: factors associated with peri-implant lesions. J Clin Periodontol. 2006;33:296-301.

20- Rupp F, Axmann D, Ziegler C, Geis-Gerstorfer J. Adsorption/desorption phenomena on pure and Teflon AF-coated titania surfaces studied by dynamic contact angle analysis. J Biomed Mater Res. 2002;62:567-78.

21- Rupp F, Scheideler L, Rehbein D, Axmann D, GeisGerstorfer J. Roughness induced dynamic changes of wettability of acid etched titanium implant modifications. Biomaterials. 2004;25:1429-38.

22- Shapiro S, Giertsen E, Guggenheim B. An in vitro oral biofilm model for comparing the efficacy of antimicrobial mouthrinses. Caries Res. 2002;36:93-100.

23- Teughels W, Van Assche N, Sliepen I, Quirynen M. Effect of material characteristics and/or surface topography on biofilm development. Clin Oral Implants Res. 2006;17(Suppl 2): 68-81.

24- Thurnheer T, Gmür R, Shapiro S, Guggenheim B. Mass transport of macromolecules within an in vitro model of supragingival plaque. Appl Environ Microbiol. 2003;69:1702-9.

25- Zijnge V, van Leeuwen MB, Degener JE, Abbas F, Thurnheer T, Gmür R, et al. Oral biofilm architecture on natural teeth. PLoS One. 2010;5:10.e9321 